\title{
Erratum
}

\section{Second primary tumor in anti-Ma1/2-positive paraneoplastic limbic encephalitis}

T. Leyhe ${ }^{1}$, R. Schüle ${ }^{1}$, F. Schwärzler ${ }^{1}$, T. Gasser ${ }^{2}$ and T. Haarmeier ${ }^{3}$

${ }^{1}$ Department of Psychiatry and Psychotherapy, University of Tübingen, Tübingen, Germany; ${ }^{2}$ Department of Neurodegenerative diseases, Hertie Institute of Clinical Brain Research, University of Tübingen, Tübingen, Germany;

${ }^{3}$ Department of General Neurology, Hertie Institute of Clinical Brain Research, University of Tübingen, Tübingen, Germany

Journal of Neuro-Oncology

DOI 10.1007/s11060-005-9052-6

The authors' affiliations were misrepresented on the title page of the original. 